\title{
Pressure-induced amorphization of noble gas clathrate hydrates
}

\author{
Paulo H. B. Brant Carvalho $\odot,{ }^{1, *}$ Amber Mace, ${ }^{2}$ Ove Andersson $\odot,{ }^{3}$ Chris A. Tulk,,${ }^{4}$ Jamie Molaison, ${ }^{4}$ \\ Alexander P. Lyubartsev, ${ }^{1}$ Inna M. Nangoi $\odot,{ }^{5}$ Alexandre A. Leitão, ${ }^{5}$ and Ulrich Häussermann $\circledast^{1}$ \\ ${ }^{1}$ Department of Materials and Environmental Chemistry, Stockholm University, SE-10691 Stockholm, Sweden \\ ${ }^{2}$ Department of Chemistry-Ångström Laboratory, Uppsala University, SE-75236 Uppsala, Sweden \\ ${ }^{3}$ Department of Physics, Umeå University, SE-90187 Umeå, Sweden \\ ${ }^{4}$ Chemical and Engineering Materials Division, Oak Ridge National Laboratory, Oak Ridge, Tennessee 37831, USA \\ ${ }^{5}$ Department of Chemistry, Federal University of Juiz de Fora, Juiz de Fora-MG, 36036-900, Brazil
}

(Received 5 October 2020; revised 19 January 2021; accepted 25 January 2021; published 17 February 2021)

\begin{abstract}
The high-pressure structural behavior of the noble gas $(\mathrm{Ng})$ clathrate hydrates $\mathrm{Ar} \cdot 6.5 \mathrm{H}_{2} \mathrm{O}$ and Xe $\cdot 7.2 \mathrm{H}_{2} \mathrm{O}$ featuring cubic structures II and I, respectively, was investigated by neutron powder diffraction (using the deuterated analogues) at $95 \mathrm{~K}$. Both hydrates undergo pressure-induced amorphization (PIA), indicated by the disappearance of Bragg diffraction peaks, but at rather different pressures, at 1.4 and above $4.0 \mathrm{GPa}$, respectively. Amorphous Ar hydrate can be recovered to ambient pressure when annealed at $>1.5 \mathrm{GPa}$ and $170 \mathrm{~K}$ and is thermally stable up to $120 \mathrm{~K}$. In contrast, it was impossible to retain amorphous Xe hydrate at pressures below $3 \mathrm{GPa}$. Molecular dynamics (MD) simulations were used to obtain general insight into PIA of Ng hydrates, from $\mathrm{Ne}$ to Xe. Without a guest species, both cubic clathrate structures amorphize at $1.2 \mathrm{GPa}$, which is very similar to hexagonal ice. Filling of large-sized H cages does not provide stability toward amorphization for structure II, whereas filled small-sized dodecahedral D cages shift PIA successively to higher pressures with increasing size of the $\mathrm{Ng}$ guest. For structure I, filling of both kinds of cages, large-sized T and small-sized D, acts to stabilize in a cooperative fashion. Xe hydrate represents a special case. In MD, disordering of the guest hydration structure is already seen at around $2.5 \mathrm{GPa}$. However, the different coordination numbers of the two types of guests in the crystalline cage structure are preserved, and the state is shown to produce a Bragg diffraction pattern. The experimentally observed diffraction up to $4 \mathrm{GPa}$ is attributed to this semicrystalline state.
\end{abstract}

DOI: 10.1103/PhysRevB.103.064205

\section{INTRODUCTION}

Clathrate hydrates are crystalline compounds consisting of a framework of tetrahedrally coordinated water molecules forming polyhedral cages which include guest species [1]. These guest species are rather diverse and can range from smaller-sized organic molecules [e.g., natural gas, tetrahydrofuran (THF)], linear and bent triatomic molecules (such as carbon dioxide and $\mathrm{H}_{2} \mathrm{~S}$ ), diatomic molecules (e.g., hydrogen, nitrogen, chlorine), to single atom noble gases (Ngs) $[2,3]$. Clathrate hydrate structures are stabilized by hydrogen bonds between water host molecules and van der Waals forces between host and guest [4]. These guest-host interactions determine the type of crystalline structure that may form: most clathrate hydrates exist in one of two cubic structures [1], termed structure I (CS-I, $P m-3 n$ space group, lattice parameter $a \approx 12 \AA$ ) [5] and structure II (CS-II, $F d-3 m$ space group, lattice parameter $a \approx 17 \AA$ ) [6,7]. Other frameworks have

\footnotetext{
*paulo.barros@mmk.su.se
}

Published by the American Physical Society under the terms of the Creative Commons Attribution 4.0 International license. Further distribution of this work must maintain attribution to the author(s) and the published article's title, journal citation, and DOI. Funded by Bibsam. been either predicted theoretically or attained experimentally at high pressures [8-15].

The two cubic framework structures feature two types of polyhedral cages each (see Fig. 1). The unit cell of CS-I contains 46 water molecules which build up two dodecahedral $5^{12}$ cages (D cages) and six tetrakaidecahedral $5^{12} 6^{2}$ cages (T cages). The unit cell of CS-II contains 136 water molecules which build up 16 dodecahedral $\mathrm{D}$ cages and 8 hexakaidecahedral $5^{12} 6^{4}$ cages (H cages) [16]. The composition of cubic clathrate hydrates is variable: sometimes the smaller-sized D cages are not occupied, or more frequently, both types of cages are occupied but not completely. In this case, typical occupancies are between 0.8 and 0.9. Several types of clathrate hydrates occur abundantly in nature and are considered future energy resources (or environmental threats) due to large amounts of stored natural gas [17]. Apart from their potential significance in gas or energy storage and transport, clathrate hydrates have been discussed for application in carbon capture and storage, water desalination, separation of ionic liquids, and food and pharmaceutical engineering [1,18-21].

Most central to clathrate hydrates is an understanding of the guest-host interactions. These interactions are particularly sensitive to density, and it has been shown that pressure is a valuable variable for probing intermolecular interaction potentials and hydration structures of guest species [22]. Investigations of cubic clathrate hydrates at high pressure 


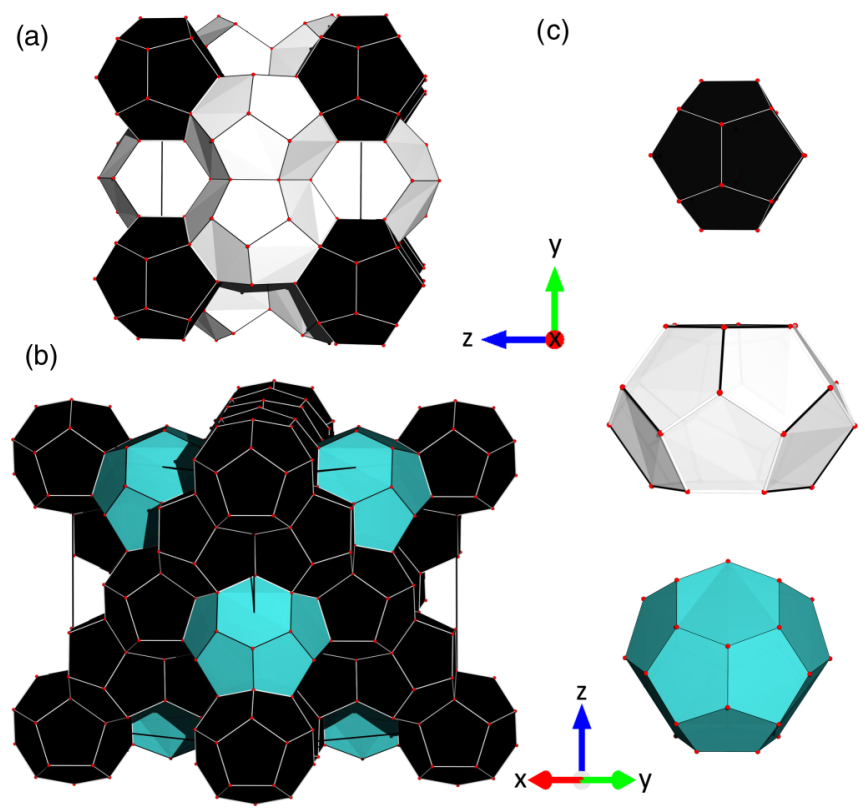

FIG. 1. Unit cell of the cubic clathrate hydrate structures. (a) CS-I (Pm-3n, $a \approx 12 \AA$ ), composed of 46 water molecules forming a hydrogen-bonded network around six large $\mathrm{T}$ cages (gray) and two small D cages (black). (b) CS-II ( $F d-3 m$ space group, lattice parameter $a \approx 17 \AA$ ), composed of 136 water molecules which build up $16 \mathrm{D}$ cages and eight $\mathrm{H}$ cages (cyan). (c) The three different cages in cubic structured clathrate hydrates: D cages (pentagon dodecahedra, $5^{12}$ ), common for both CS-I and CS-II; T cages (tetrakaidecahedra, $5^{12} 6^{2}$ ), large cages in CS-I; and $\mathrm{H}$ cages (hexakaidecahedra, $5^{12} 6^{4}$ ), large cages in CS-II (and largest among all). T cages are represented in (c) with their two different types of pentagon faces: those constituting $\mathrm{H}$ cages only (black edges), and those constituting both $\mathrm{H}$ and $\mathrm{D}$ cages (white edges). Red spheres on cage vertexes represent oxygen atoms.

(in the gigapascal regime) and close to room temperature (260-280 K) have led to the observation of new types of hydrate frameworks, both of clathratic nature and filled-ice structures [8,9]. The high-pressure hydrates have typically more guest-rich compositions than the original cubic clathrate hydrates since they follow from decomposition of the latter. The structure of high-pressure hydrates and phase diagrams are discussed in the literature $[8,9,12-15,22-26]$. When compressed at lower temperatures (below $135 \mathrm{~K}$ ), cubic clathrate hydrates exhibit instead a sharp, first-order-like transition to an amorphous state, analogous to pure water's high-density amorphous (HDA) ice, which is obtained when pressurizing hexagonal ice $I_{h}$ at similarly low temperatures [26]. Since the tetrahedral nearest neighbor arrangement of water molecules in the host structure of cubic clathrate hydrates is identical to ice $I_{h}$ and the concentration of the guest is rather small, their pressure-induced amorphization (PIA) should follow the same or a very similar mechanism. However, since examples of cubic clathrate hydrates displaying PIA are rather limited, this is still not clear. Likewise, structural and physical properties of the amorphous forms are largely unexplored.

Noble gas clathrate hydrates were targeted early on as model systems for the statistical thermodynamic analysis of clathrate hydrates [4]. This is because monoatomic Ngs are distinguished in their isotropic interactions with the hosting water cage, which are easy to model, and by providing a homologous series of increasingly larger (and heavier) guests. Studies can then systematically address effects of guest size and cage occupancy to clathrate hydrate stability. Consequently, $\mathrm{Ng}$ clathrate hydrates also represent ideal model systems for studying the PIA phenomenon. We selected Xe and Ar clathrate hydrate, which adopt the CS-I and CS-II structure, respectively, as prototypical systems. Their composition has been reported as $\mathrm{Ar} \cdot 6.5 \mathrm{H}_{2} \mathrm{O}$ (D $89 \%$ filled and $\mathrm{H}$ $83 \%$ ) and $\mathrm{Xe} \cdot 7.2 \mathrm{H}_{2} \mathrm{O}$ (D 76\% filled and $\mathrm{T} 81 \%$ ) $[6,23,24]$. The near room temperature high-pressure behavior is also well studied [9]. With increasing pressure, CS-II Ar hydrate (AH) transforms successively into a hexagonal, tetragonal, and orthorhombic clathrate structure variant. The latter is stable up to $6 \mathrm{GPa}$, after which decomposition into solid Ar and ice VII occurs [8-11]. CS-I Xe hydrate (XH) was found stable up to about 1.7 GPa. At higher pressure, the phase undergoes a transition to the hexagonal clathrate structure (which can be recovered to ambient pressure at $77 \mathrm{~K}$ in the slightly modified HS-I structure) [12]. Above $2.9 \mathrm{GPa}$, the hexagonal clathrate structure decomposes into solid Xe and ice VII [13-15]. With respect to high-pressure investigations at low temperatures (i.e., below $135 \mathrm{~K}$ ), nothing is known about Ar clathrate hydrate, whereas CS-I XH was included in the seminal study by Handa et al. [27]. These authors performed piston cylinder experiments on CS-II THF, $\mathrm{SF}_{6}$ hydrates, and CS-I XH at $77 \mathrm{~K}$ and up to $1.7 \mathrm{GPa}$. It was found that the CS-II type clathrate hydrates amorphized, whereas XH did not undergo any transformation in the applied pressure range. However, recent potential energy minimization calculations (using empirical potentials) considering fully occupied CS-I Ng hydrate for $\mathrm{Ng}=\mathrm{Ar}, \mathrm{Kr}$, and Xe predicted PIA at 1.8, 2.6, and $4 \mathrm{GPa}$, respectively (referring to $0 \mathrm{~K}$ ) [28].

In this paper, high-pressure neutron powder diffraction (NPD) experiments were carried out on CS-II AH and CS-I $\mathrm{XH}$ for pressures from 0.1 to $5 \mathrm{GPa}$ at $95 \mathrm{~K}$. Both clathrate hydrates were found to collapse into an amorphous state. The PIA behavior was then examined with molecular dynamics (MD) simulations, addressing the complete series of $\mathrm{Ng}$ atoms, Ne through Xe.

\section{EXPERIMENTAL DETAILS AND METHODS OF ANALYSIS}

\section{A. NPD experiments}

A range of NPD experiments involving several samples of $\mathrm{Ar}$ and Xe clathrate hydrates were conducted at the SNAP beamline at the Spallation Neutron Source (SNS), Oak Ridge National Laboratory (ORNL), USA. All samples were synthesized onsite on a setup put in place at SNAP. Data from 10 experiments were combined in this paper, and details to each sample and experiment can be found in the Supplemental Material (SM) [29].

\section{B. Synthesis of XH}

Fully deuterated CS-I Xe clathrate hydrate was synthesized similarly to the procedure described in Ref. [27]. Finely ground and sieved ( $<45 \mu \mathrm{m}$ particles) $\mathrm{D}_{2} \mathrm{O}$ ice $(99.9 \%$ deuterated, Sigma Aldrich) was prepared and transferred to a cold 
pressure vessel, like the gas cell used for the synthesis of ${ }^{36} \mathrm{AH}$ as in Ref. [6]. The vessel was sealed and filled with $\sim 50$ bar of precooled Xe and kept in a freezer at $250 \mathrm{~K}$ for $>10$ days. The precursor ice $I_{h}$ was still found present in all samples $(\sim 10$ to $40 \%$ of ice contaminant), possibly due to a synthesis time that was too short. Xe hydrate samples were recovered and stored in $\mathrm{LN}_{2}$ prior to NPD experiments.

\section{Synthesis of AH}

Fully deuterated CS-II clathrate hydrate of Ar was synthesized following the procedure described in Ref. [6]. Finely ground $\mathrm{D}_{2} \mathrm{O}$ ice (99.9\% deuterated, Sigma Aldrich) was prepared and transferred to a cold (below freezing) pressure vessel containing stainless steel ball bearings. The vessel was sealed and filled with 150 bar of precooled Ar. The vessel was then intermittently tumbled for $>10$ days in a freezer at $250 \mathrm{~K}$, providing refreshed grain surfaces for a complete clathrate formation. The complete conversion of ice $I_{h}$ to the CS-II clathrate was confirmed by powder x-ray diffraction on a Panalytical X'Pert Pro diffractometer using the Anton Paar TTK 450 Low Temperature Chamber. Ar hydrate samples were recovered and stored in $\mathrm{LN}_{2}$ prior to NPD experiments.

\section{In Situ Time-of-Flight NPD}

Time-of-flight NPD experiments were performed in the temperature range of $95-220 \mathrm{~K}$ using a standard VX Paris-Edinburgh pressure cell with $\mathrm{LN}_{2}$-cooled stainless-steel anvils, which can reach up to $10 \mathrm{GPa}$ at temperatures as low as $77 \mathrm{~K}$. Samples were loaded at $\mathrm{LN}_{2}$ temperature in null-scattering TiZr gaskets, with lead powder added as a pressure marker. The gaskets were pressurized to $\sim 0.1 \mathrm{GPa}$ upon loading to stabilize the samples, which warmed up to about $170 \mathrm{~K}$ during transferring of the pressure cell to the cryostat, where they were cooled to $95 \mathrm{~K}$. For all samples, a longer $(\sim 2 \mathrm{~h})$ starting collection of neutron diffractograms at the crystalline stage was done, allowing the refinement of the clathrate hydrate unit cells and identification of contaminants, if any. Pressurization was done in steps of 0.1-0.2 GPa, followed by data collection during $15 \mathrm{~min}$ to up to several hours. Samples were compressed until their full amorphization could be observed by the disappearance of all their Bragg diffraction peaks. In addition, vanadium powder and an empty gasket were measured for intensity normalization and for background correction, respectively. Masking of the detector banks was done during data treatment at higher-pressure steps due to partial shading of the detectors by the anvil and press materials.

\section{E. MD Modeling}

Simulation cells were constructed using the GenIce software [30], which places oxygen atoms at their crystallographic sites in the cubic unit cell, randomizes the distribution of hydrogen atoms constrained by the Bernal-Fowler ice rules, and chooses the configuration with zero (minimum) net polarization. CS-II supercells consist of $2 \times 2 \times 2$ periodically replicated cubic unit cells consisting of $1088 \mathrm{H}_{2} \mathrm{O}$ molecules with $\sim 34 \AA$ initial cell dimensions. CS-I supercells consist of $3 \times 3 \times 3$ periodically replicated cubic unit cells consisting of $1242 \mathrm{H}_{2} \mathrm{O}$ molecules with $\sim 36 \AA$ initial cell dimensions. The guest atoms ( $\mathrm{Ne}, \mathrm{Ar}, \mathrm{Kr}, \mathrm{Xe})$ were randomly distributed fulfilling the following compositions: (case i) full occupancies, (case ii) only small (D) cages filled; case (iii) only large (CS-I: T, CS-II: H) cages filled; (case iv, Ne only) CS-II with only $\mathrm{H}$ cages doubly occupied, and full occupancy of D cages plus $\mathrm{H}$ cages doubly occupied.

The PIA was modeled with isotropic (NPT) MD simulations in the LAMMPS software [31]. To describe the interatomic interaction during the MD simulations, the TIP4P/Ice [32] and UFF [33] force fields were used for ice and Ngs, respectively, and the Lorentz-Berthelot mixing rules were applied. The Nose-Hoover barostat algorithm with the Melchionna modification [34] was employed to control the temperature and pressure of the systems. The simulations were carried out at $95 \mathrm{~K}$, and the pressure was subsequently increased from 1 to $3 \mathrm{GPa}$ in steps of $0.05 \mathrm{GPa}$. At each pressure step, the system was first incremented gradually over $2 \mathrm{~ns}$; the system was then equilibrated for $5 \mathrm{~ns}$, followed by a further $1 \mathrm{~ns}$ simulation during which statistics for the densities and radial pair distribution functions (PDFs) were collected. The simulation time step was $1.0 \mathrm{fs}$, and barostat relaxation time was set to 2000 time steps.

Snapshots (single frame in a simulation) were taken from $100 \%$ CS-I XH and 100\% CS-II AH MD calculations at pressure steps just before (CS-I: $2.4 \mathrm{GPa}$; CS-II: $1.4 \mathrm{GPa})$ and immediately after collapse (CS-I: 2.6 and $2.9 \mathrm{GPa}$; CS-II: $1.7 \mathrm{GPa}$ ). MD supercells (i.e., $2 \times 2 \times 2 \mathrm{CS}$-II and $3 \times$ $3 \times 3$ CS-I unit cells) were doubled along each axis (now corresponding to $4 \times 4 \times 4$ CS-II and $6 \times 6 \times 6$ CS-I cells) for simulating neutron diffraction patterns by the XaNSoNS software [35].

\section{RESULTS AND DISCUSSION}

\section{A. PIA of CS-I XH and CS-II AH}

Although the synthesis of $\mathrm{Xe}$ and Ar clathrate hydrate followed established protocols [6,27], XH samples showed various amounts of ice $I_{h}$, whereas AH could be prepared virtually ice-free. For this paper, the presence of ice was deemed to not interfere with obtained results. The composition of the hydrates was assumed to be as earlier determined by Flacau et al. [23], $\mathrm{Xe} \cdot 7.2 \mathrm{H}_{2} \mathrm{O}$ (D 76\% filled and $\mathrm{T} 81 \%$ ), and Yang et al. and Brant Carvalho et al. [6,24], $\mathrm{Ar} \cdot 6.5 \mathrm{H}_{2} \mathrm{O}$ (D 89\% filled and $\mathrm{H} 83 \%$ ). Figure 2 shows initial neutron diffraction patterns of Xe and Ar clathrate hydrate samples at $95 \mathrm{~K}$ and about $0.1 \mathrm{GPa}$ (experiments AH5 and XH2, respectively, see SM for details [29]). For fitting the diffraction data by Rietveld refinement, the structures from Ikeda et al. [36] and Brant Carvalho et al. [6] were used as the starting models for $\mathrm{Xe}$ and $\mathrm{Ar}$ clathrate hydrate, respectively. Refined parameters included background and peak profile terms, hydrate/ice content ratio and unit cell parameters. As a general procedure, the samples were first isothermally compressed at $95 \mathrm{~K}$ until a structural change, i.e., amorphization, occurred. Afterwards, various $\mathrm{p}, \mathrm{T}$ pathways were pursued to check the reversibility of observed transitions. These pathways are depicted in Fig. 3, and below, we summarize findings (see SM for details to specific AH and XH experiments [29]). 

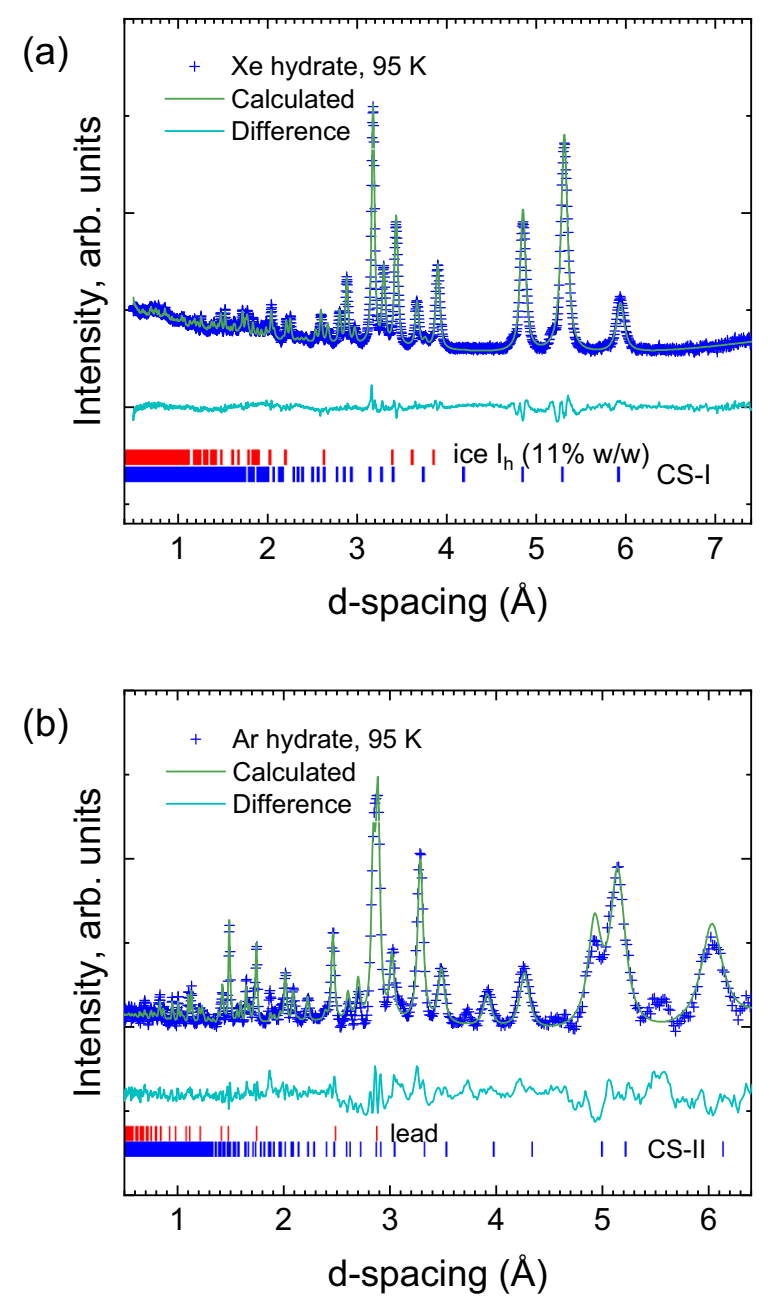

FIG. 2. Experimental neutron powder diffraction (NPD) profiles of (a) CS-I Xe and (b) CS-II Ar clathrate hydrate. The diffractograms were measured at $95 \mathrm{~K}$ and at the lowest pressure point $0-0.1 \mathrm{GPa}$. Profiles obtained upon Rietveld refinement are shown as solid lines. The difference curves are plotted beneath. Tick marks show Bragg positions of allowed reflections for the fitted phases. Xe hydrate: $a=11.8662(6) \AA, R_{\mathrm{wp}}=3.73 \%, \operatorname{GoF}\left(\chi^{2}\right)=3.04$. Ar hydrate: $a=$ $17.040(3) \AA, R_{\mathrm{wp}}=1.37 \%, \operatorname{GoF}\left(\chi^{2}\right)=2.90$.

During compression, the hydrate phases were consistent with the structure in the literature, despite the peak broadening caused by strain effects. CS-I XH was observed to be stable at $95 \mathrm{~K}$ up to $4.0 \mathrm{GPa}$, and CS-II $\mathrm{AH}$ up to $1.4 \mathrm{GPa}$. Beyond these pressures, both hydrates transformed into amorphous states. The PIA was characterized by the complete disappearance of all Bragg diffraction peaks of a given phase. A representative evolution of the diffraction patterns with pressure is shown in Fig. 4. The behavior of CS-II AH appears very similar to earlier investigated CS-II hydrates (i.e., THF, 1,3-dioxolane, cyclobutanone, acetone [37], $\mathrm{SF}_{6}{ }^{27}$ ), for which amorphization was observed in a pressure range of $1.2-1.6 \mathrm{GPa}$ at similar temperatures. This is at slightly higher pressures than the pressure of the ice $I_{h}$-to-HDA transition $(\sim 1 \mathrm{GPa})$ [26]. In contrast, the PIA of CS-I XH occurred at considerably higher pressures. There is only one more example of a CS-I hydrate

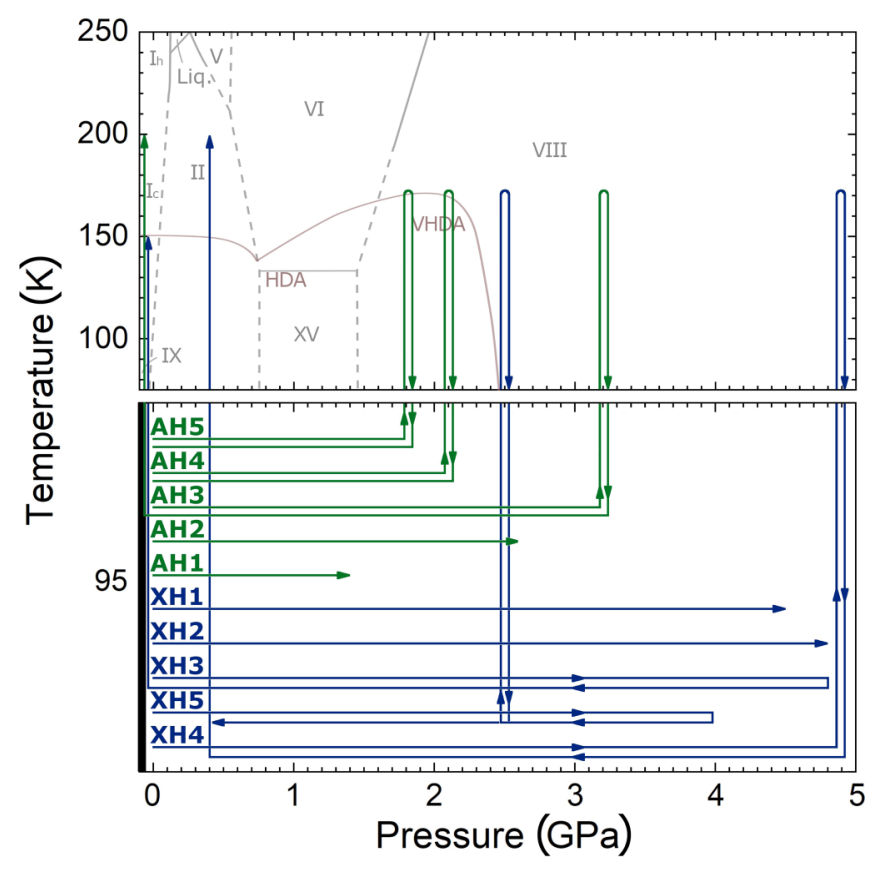

FIG. 3. Experimental pathways followed in this paper. The phase diagram of pure ice (from Salzmann, Ref. [54]) is underlaid as background. Ar hydrate (AH) experiments (green), AH1: Compression to PIA (1.4 GPa); AH2: Compression to 2.6 GPa; AH3: Compression to PIA and up to $3.15 \mathrm{GPa}$, temperature cycling to $170 \mathrm{~K}$, cooling, decompression, and heating to $200 \mathrm{~K}$ at ambient pressure; $\mathrm{AH} 4$ : Compression to $2 \mathrm{GPa}$, temperature cycling to $170 \mathrm{~K}$, cooling, and decompression; AH5: Compression to $1.9 \mathrm{GPa}$, temperature cycling to $170 \mathrm{~K}$, cooling, and decompression. Xe hydrate $(\mathrm{XH})$ experiments (navy blue), XH1: Compression to $4.5 \mathrm{GPa}$; XH2: Compression to $4.8 \mathrm{GPa}$; XH3: Compression to $5.1 \mathrm{GPa}$, decompression to atmospheric pressure, and heating to $150 \mathrm{~K}$; XH4: Compression to 4.9 $\mathrm{GPa}$, temperature cycling to $170 \mathrm{~K}$, cooling, decompression to $0.4 \mathrm{GPa}$, and heating to $200 \mathrm{~K}$; XH5: Compression to $4 \mathrm{GPa}$, decompression to $2.5 \mathrm{GPa}$, heating to $170 \mathrm{~K}$, and cooling back to $95 \mathrm{~K}$, decompression to $0.48 \mathrm{GPa}$.

which has been studied by low temperature compression: $\mathrm{CH}_{4}$ hydrate was pressurized to $3.2 \mathrm{GPa}$ at $100 \mathrm{~K}$, resulting in an amorphous sample. Although the pressure for PIA was not precisely determined in this experiment, it indicates that CS-I hydrates amorphize at higher pressures than CS-II hydrates.

To investigate the reversibility of PIA, the amorphous hydrates were then brought back to atmospheric pressure at temperatures near $100 \mathrm{~K}$. Unlike HDA ice (but like THF and other CS-II hydrates), both amorphous clathrate hydrates recrystallize upon low temperature decompression. In another experiment, we temperature cycled the amorphized hydrates to $\sim 170 \mathrm{~K}$ at a pressure above the amorphization pressure, following the procedure from Suzuki [38] and Bauer et al. [39]. This procedure has been shown to produce a recoverable, denser amorphous form for several CS-II hydrates [38-41], which correlates to the "very HDA" form of water ice [42]. Indeed, after temperature cycling, amorphous $\mathrm{AH}$ did not recrystallize when recovered to ambient pressure at $95 \mathrm{~K}$ (experiments AH3-5, SM [29]). Upon heating the recovered amorph, recrystallization was seen at $120 \mathrm{~K}$. At $150 \mathrm{~K}$, the 

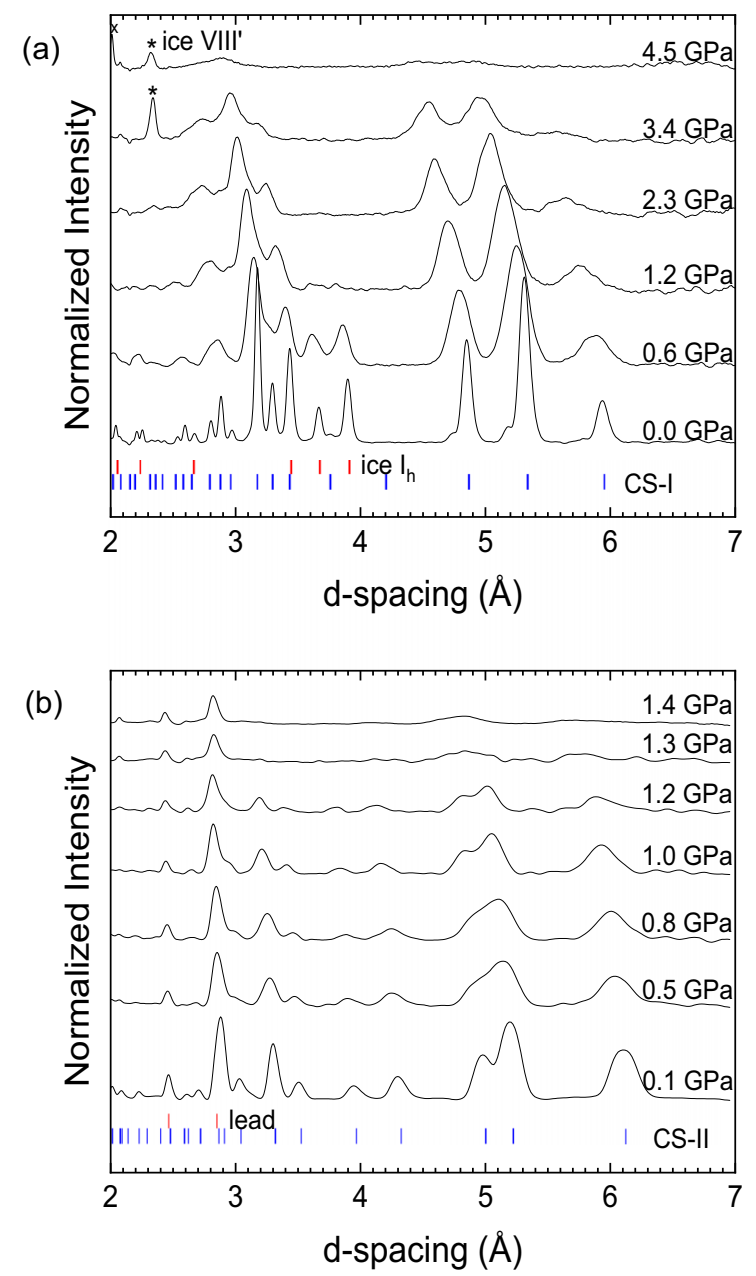

FIG. 4. Evolution of the neutron powder diffraction (NPD) profiles of (a) CS-I Xe and (b) CS-II Ar hydrate upon compression. Tick marks show Bragg positions of allowed reflections for the fitted phases. Stars mark peaks from ice (111 reflection of ice VII or 112 reflection of ice VIII), which can be seen in (a) after recrystallization of high-density amorphous (HDA) above $2.5 \mathrm{GPa}$. Cross marks the 110 peak of iron as the steel anvil shades part of the beam at high pressures. Patterns were treated for background (including diffuse scattering from the samples) and smoothed for clarity.

clathrate hydrate sample decomposed to Ar and ice $I_{h}$, in agreement with earlier observations [43]. In contrast, amorphous XH recrystallized in all attempts of recovery from high pressures. As a matter of fact, crystalline features from the CS-I phase were already observed at $\sim 3 \mathrm{GPa}$ on decompression (Fig. S1, SM [29]). The recrystallized XH samples were carefully investigated for their ice content. As a reminder, XH samples contained various amounts of $I_{h}$, which upon compression at $95 \mathrm{~K}$ forms HDA at around $1 \mathrm{GPa}$ and then recrystallizes as ice VIII' above 2.5 GPa [see Fig. 4(a)] [44]. On pressure release, $I_{h}$ reforms. We did not find a significantly changed volume fraction of $I_{h}$ with respect to the starting samples (differences were below 4\%), which indicates that recrystallized XH and most likely also its amorphous form have the same composition. We infer that amorphous $\mathrm{AH}$ and $\mathrm{XH}$ behave differently with respect to their recoverability to ambient pressure, but we cannot entirely rule out that crystalline
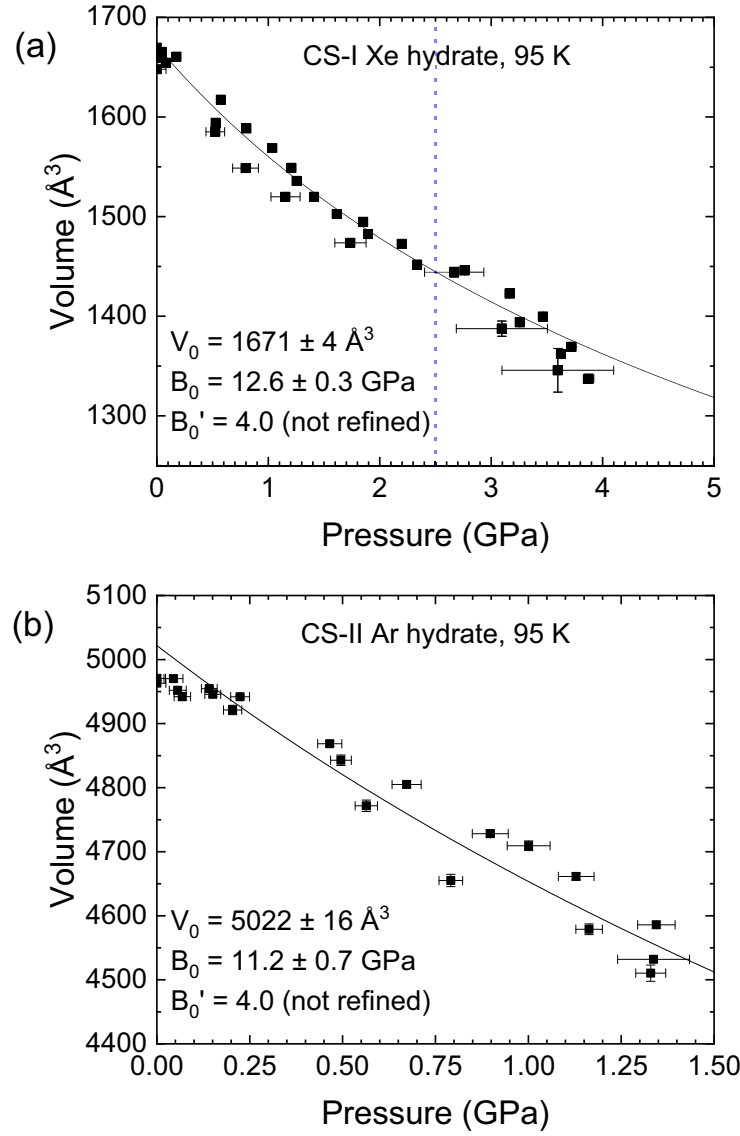

FIG. 5. Equations of state at $95 \mathrm{~K}$ of (a) Xe hydrate and (b) Ar hydrate. The line is a fit of the Murnaghan equation $V_{0} / V=$ $\left[p\left(B^{\prime} / B\right)+1\right]^{1 / B^{\prime}}$ with $V_{0}, B$, and $B^{\prime}$ being the volume at zero pressure, the bulk modulus, and its first pressure derivative, respectively. The dashed line in (a) marks a transition to a semicrystalline state, as suggested from molecular dynamics (MD) simulations.

domains of ice VIII' influence the recrystallization behavior of amorphous $\mathrm{XH}$.

\section{B. Equation of states of CS-I XH and CS-II AH and analysis of their PIA behavior}

The diffraction patterns provide the evolution of the hydrate lattices with increasing pressure, which were used to calculate the equation of state (EoS) of the two hydrates at $95 \mathrm{~K}$. For this, we used the simple Murnaghan relation $V_{0} / V=\left[p\left(B^{\prime} / B\right)+1\right]^{1 / B^{\prime}}$, with $V_{0}, B$, and $B^{\prime}$ being the volume at zero pressure, the bulk modulus, and its first pressure derivative, respectively [45]. (Note that strictly the Murnaghan relation is only valid for hydrostatic conditions, which are not fulfilled in our experiments.) The obtained EoS are shown in Figs. 5(a) and 5(b). The extrapolated ambient pressure lattice parameters are within experimental error of those reported in the literature: CS-I XH: $a_{0}=11.87(52) \AA$; CS-II AH: $a_{0}=17.12(84) \AA$ [46]. The bulk modulus for CS-I XH is $12.7(3) \mathrm{GPa}$ in the $0-4 \mathrm{GPa}$ pressure range, and the one for CS-II AH is 11.2(7) GPa in the 0-1.4 GPa range. We note that a previous determination of the bulk modulus for $\mathrm{XH}$ at room temperature and for the pressure interval of its stability at this 


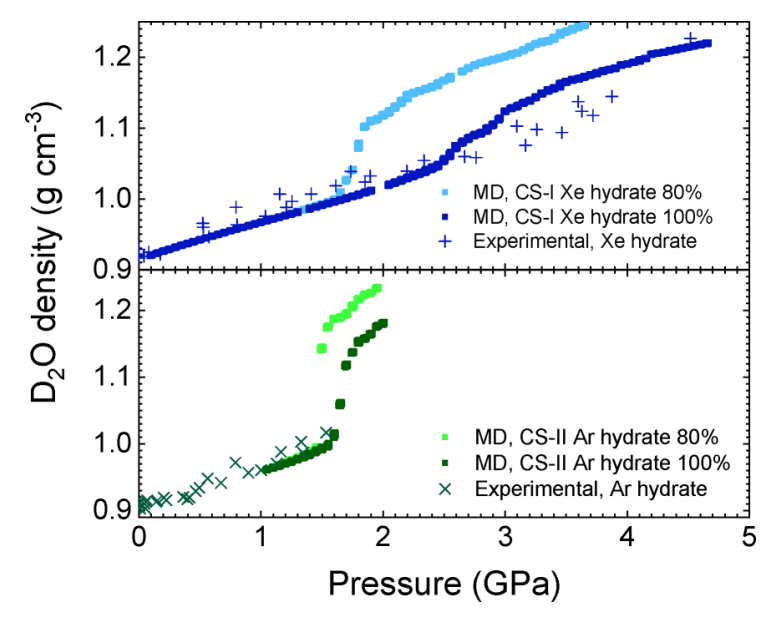

FIG. 6. Density vs pressure comparison from neutron powder diffraction (NPD) experiments and molecular dynamics (MD) simulations. CS-I Xe hydrate (top) and CS-II Ar hydrate (bottom). MD data were rescaled for $\mathrm{D}_{2} \mathrm{O}$, from simulations of cage occupancies ideally fully occupied (100\%) and approaching the experimental composition: $80 \%$ of $\mathrm{D}$ and $\mathrm{T}$ cages filled in Xe hydrate, $80 \%$ of $\mathrm{D}$ and $\mathrm{H}$ cages filled in Ar hydrate.

temperature 0-1.5 GPa yielded a value of 9.3(4) GPa [15,47] (whereas the one of $\mathrm{AH}$, in the pressure range $0-0.7 \mathrm{GPa}$, does not seem to be determined). Manakov et al. recently showed that the bulk moduli of CS-I and CS-II hydrates at room temperature, in most cases, were close to each other with $B=9(2) \mathrm{GPa}$ and are mainly determined by the elasticity of the hydrogen-bonded framework [47].

Figure 6 shows the MD calculated density for $\mathrm{Xe}$ and $\mathrm{Ar}$ clathrate hydrate, rescaled for $\mathrm{D}_{2} \mathrm{O}$, at $95 \mathrm{~K}$ as a function of pressure. For the simulations, we considered two scenarios, fully occupied cages and a random $80 \%$ occupancy for both types of cages. The latter scenario should correspond closely to the experimental composition. The compressibility of the crystalline forms is in good agreement with the experimental values. For Ar clathrate hydrate, the calculated density increases discontinuously above 1.4 and $1.6 \mathrm{GPa}$ for the 80 and $100 \%$ occupied cases, respectively, which signals the onset of amorphization. This is in very good agreement with the experimentally observed amorphization pressure. We note that full occupancy of cages slightly increases the amorphization pressure, i.e., stabilizes the CS-II clathrate, and we will return to this subject later.

At first sight, the MD calculated and experimentally observed compression behavior for CS-I XH do not seem to agree well. The MD simulations predict amorphization above 1.6 and $2.5 \mathrm{GPa}$ for the 80 and $100 \%$ occupied cases, respectively. In the experiment, however, Bragg diffraction is clearly present up to at least $4 \mathrm{GPa}$ [cf. Fig. 4(a)]. At the same time, the MD predicted transition appears sluggish, especially for the fully occupied case for which full densification of the amorphous form is not attained below $3 \mathrm{GPa}$. The reason for the sluggish transition and the discrepancy to the experimentally observed amorphization may be found in the PDFs.

Figure 7 depicts the evolution of PIA by comparing the PDFs for the fully occupied clathrate models. The rather abrupt transformation for $\mathrm{AH}$ at $1.7 \mathrm{GPa}$ is seen with a nonzero $g_{\mathrm{OO}}(r)$ after the first coordination shell, $r>3 \AA$ (corresponding to four water molecules), and the liquidlike, rather featureless shape of $g_{\mathrm{OO}}(r)$ beyond $4 \AA$ [Fig. 7(a)]. This coincides with the collapse of cages as seen with the $g_{\mathrm{OAr}}{ }^{\mathrm{H}}(r)$ and $g_{\mathrm{OAr}} \mathrm{D}(r)$. For the crystalline structure, before the collapse, the O-Ar contacts for the large $\mathrm{H}$ and small $\mathrm{D}$ cage are at about 4 and $3.7 \AA$, respectively, whereas after the collapse, the contacts in both cages are at about $3.4 \AA$ [Figs. 7(b) and 7(c)]. The integrated coordination numbers for $\mathrm{Ar}^{\mathrm{H}}$ and $\mathrm{Ar}^{\mathrm{D}}$ attain a similar value out to $4.5 \AA$, around 19 , which indicates a similar hydration structure for both kinds of Ar atoms. For $\mathrm{XH}$, the corresponding PDFs [shown in Figs. 7(d)-7(f)] reveal that PIA is a more continuous process, as already indicated in the density vs pressure behavior [cf. Fig. 6]. Molecular dynamics suggest amorphization at $2.5 \mathrm{GPa}$, which coincides with the appearance of a nonzero $g_{\mathrm{OO}}(r)$ after the first coordination shell (in the range $r=3-3.5 \AA$ ), Fig. 7(d). However, the change of the $\mathrm{XH} g_{\mathrm{OO}}(r)$ at $2.5 \mathrm{GPa}$ is not as abrupt as the one of the AH at 1.7 GPa [cf. Fig. 7(a)]. With increasing pressure, changes remain smooth. Specifically, the oscillating, crystalline feature after the first coordination shell is maintained up to $3 \mathrm{GPa}$. Eventually, at $4 \mathrm{GPa}$, the $\mathrm{XH} g_{\mathrm{OO}}(r)$ resembles that of $\mathrm{AH}$ after amorphization (i.e., at $1.7 \mathrm{GPa}$ ). The structural change at $2.5 \mathrm{GPa}$ is most clearly seen from the evolution of $g_{\text {OXe }} \mathrm{T}^{\mathrm{T}}(r)$ for which the first maximum shifts discontinuously from 3.8 to $3.7 \AA$ [Fig. 7(e)], indicating the collapse of large cages. At the same time, the coordination number for $\mathrm{Xe}^{\mathrm{T}}$ remains very similar to that of the crystalline structure, and further changes only weakly up to $2.8 \mathrm{GPa}$. For the small (D) cages, O-Xe contacts are at $\sim 3.65 \AA$ in the crystalline structure [Fig. 7(f)], which suggests repulsive interactions since the van der Waals radii for Xe and the $\mathrm{O}$ atom are 2.16 and $1.59 \AA$, respectively [48]. The $g_{\text {OXe }} \mathrm{D}(r)$ functions look almost unchanged in the pressure range 2.33.0 GPa, which indicates that Xe atoms in the small cages maintain the ordered hydration environment of the crystalline structure.

From the PDF analysis, it can be inferred that PIA for $\mathrm{XH}$ proceeds very differently from $\mathrm{AH}$. We interpret the change observed at $2.5 \mathrm{GPa}$ as a transition to a semicrystalline state which is characterized by intact small cages and "collapsed" large cages in which $\mathrm{Xe}^{\mathrm{T}}$ attains a disordered hydration environment but retains the coordination number from the crystalline structure. The further transition into the amorphous state is then a continuous process, which is completed above $4 \mathrm{GPa}$. During this process Xe atoms keep a quasi-ordered (periodic) arrangement which produces Bragg diffraction peaks. This picture is supported from MD snapshots, which are compiled in the SM [Fig. S2(a)] [29]. Note that, in contrast, during the discontinuous amorphization of CS-II AH, Ar atoms are moved significantly and irregularly from their crystalline equilibrium positions [Fig. S2(b)].

We then reexamined the diffraction patterns of $\mathrm{XH}$ [cf. Fig. 4(a)] with respect to the structural progression suggested by MD and included the diffuse scattering background, which should reveal an increasingly amorphized sample. A collection of these patterns is shown in Fig. 8 together with simulated diffraction patterns from MD snapshots at corresponding pressures. Indeed, one can notice an increased background when going from the 2.3 to the $3.4 \mathrm{GPa}$ 


\section{CS-II Ar hydrate $100 \%$ filled}

(a)
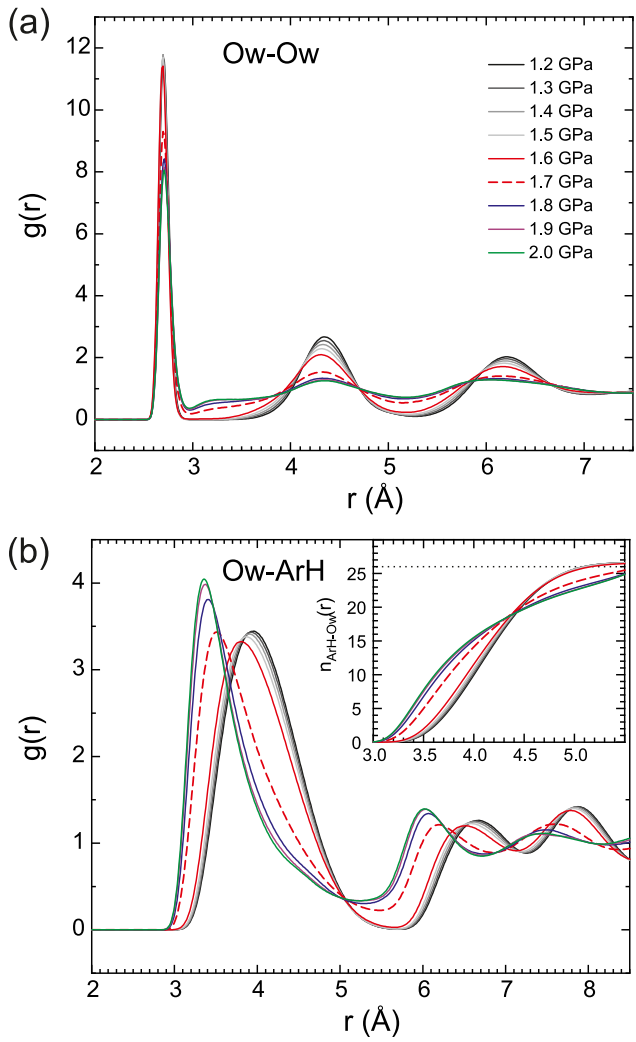

(c)

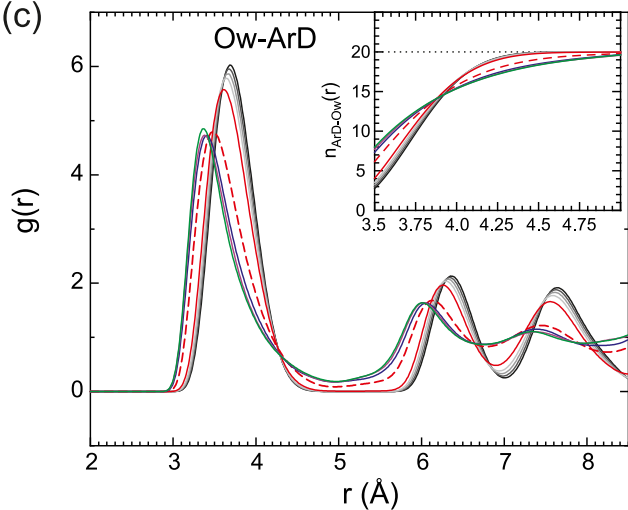

CS-I Xe hydrate $100 \%$ filled
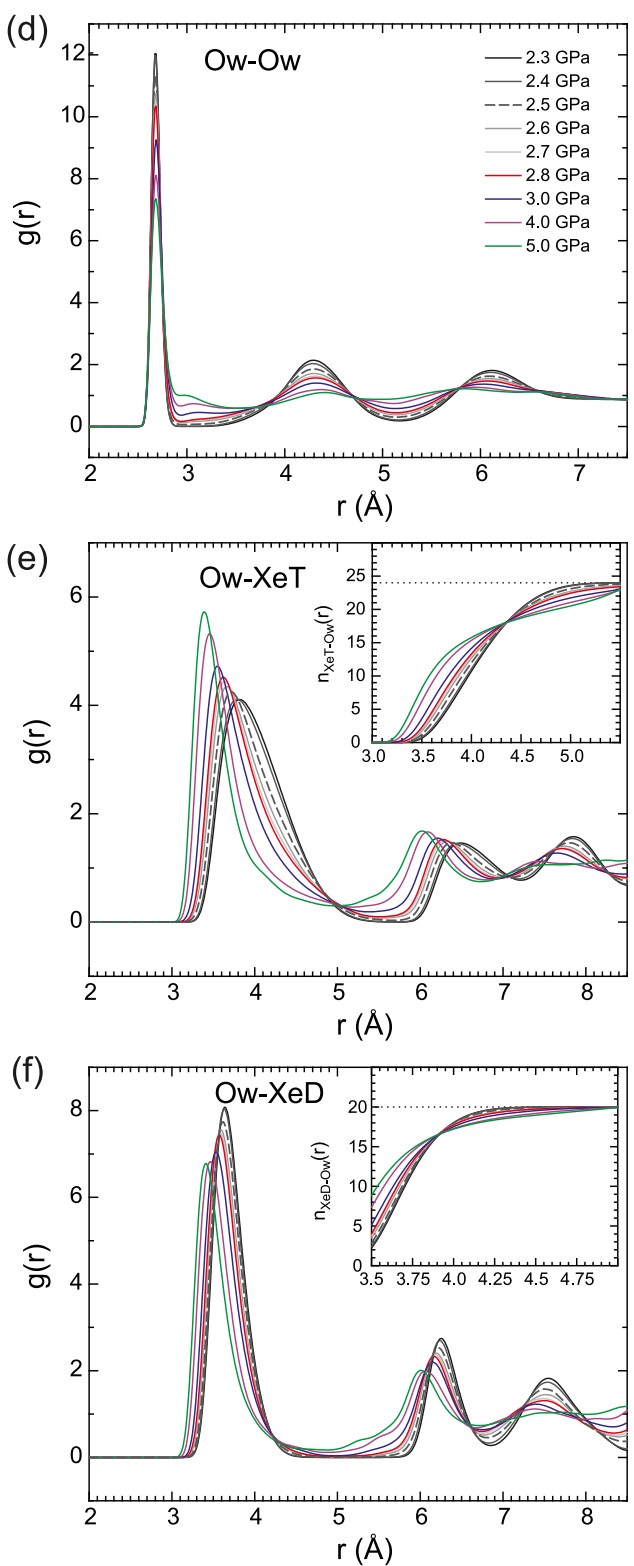

FIG. 7. Pressure dependence of the pair-distribution functions from the simulated molecular dynamics (MD) Ar and Xe hydrate systems at 95 K. (a) and (b) Oxygen-oxygen pair distribution functions (PDFs) from the water structures. (c)-(f) O-Ng PDFs with respect to guests inside small (D) and large ( $\mathrm{T}$ and $\mathrm{H}$ ) cages. The PDFs after transitions (crystalline $\rightarrow$ amorphous and crystalline $\rightarrow$ semicrystalline for Ar and Xe, respectively) are highlighted with broken lines. The insets in (b), (c) and (e), (f) show coordination numbers for $\left(\mathrm{Ar}^{\mathrm{H}}, \mathrm{Ar}^{\mathrm{D}}\right)$ and $\left(\mathrm{Xe}^{\mathrm{T}}, \mathrm{Xe}^{\mathrm{D}}\right)$, respectively.

pattern, and one may still discern weak Bragg features in the $4.5 \mathrm{GPa}$ pattern. We note the good agreement with the simulated patterns, confirming that semicrystalline and a progressively amorphous state still give rise to Bragg diffraction peaks.

Figure 9 shows the major structural features of $\mathrm{AH}$ and $\mathrm{XH}$ [Figs. 9(a) and 9(b)] prior and after their amorphization and transition to the semicrystalline state, respectively [Figs. 9(c) and 9(d)]. It is seen that the densification of clathrate hydrate affects especially the larger hexagon rings of the H (CS-II) and $\mathrm{T}$ (CS-I) cages, whereas pentagon rings of the D cages maintain a rigid structure. A similar observation was made for CS-II THF hydrate with only H cages occupied [49]. The de- formation of six-membered rings indicates (or triggers) onset of amorphization or transition. Concomitant with the onset of discontinuous densification, the $\mathrm{Ng}$ guest vibrational motion appears substantially hindered. Actual amorphization of CS-II $\mathrm{AH}$ is then characterized by an increasing distortion of pentagon rings, leading to the successive "collapse" of D cages [Fig. 9(c)]. At the same time, the coordination around both types of Ar atoms rearranges into that of the final amorphous state, which means that the hydration shells for the originally differently coordinated Ar guests even out. In contrast, apart from disordering, Xe guest atoms retain the hydration shell from the crystalline structure in the semicrystalline state up to 3 GPa [Fig. 9(d)]. 


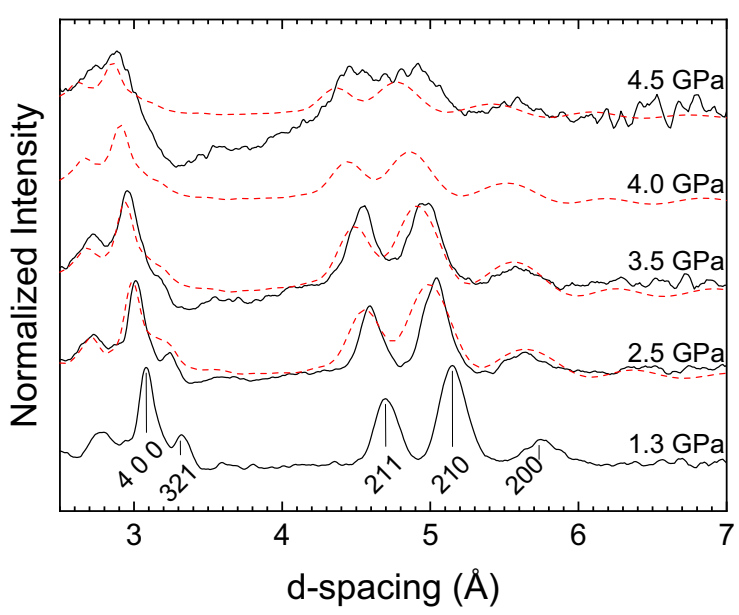

FIG. 8. Neutron powder diffraction (NPD) patterns showing the structural progression of CS-I Xe hydrate with pressure in experiments and molecular dynamics (MD) snapshots. Black lines represent experimental data (experiment XH2, SM [29]). Red-dotted lines represent simulated patterns from MD snapshots. Patterns show no sudden change in the long-range structure, and the final pattern with still crystalline features is in good agreement with experimental data.

\section{Generalized PIA behavior for Ng clathrate hydrates from MD studies}

We extend our study by looking generally at PIA behavior for $\mathrm{Ng}$ clathrate hydrates from MD studies. We first compare the $\mathrm{Ng}$ series for $\mathrm{Ne}$ to $\mathrm{Xe}$ for the two cubic structures, assuming fully occupied cages, i.e., $8 \mathrm{Ng} \cdot 46 \mathrm{H}_{2} \mathrm{O}$ for CS-I and $24 \mathrm{Ng} \cdot 136 \mathrm{H}_{2} \mathrm{O}$ for CS-II. The density-pressure relationships, referring to $95 \mathrm{~K}$, and also including the empty clathrate structures, are shown in Fig. 10.

The empty clathrates amorphize at about $1.2-1.25 \mathrm{GPa}$, irrespective of the kind of cubic structure. This transition pressure is very similar to that of $I_{h}$, which has already been pointed out by Handa et al. [27]. The $I_{h}$-to-HDA transition from MD calculations is typically seen at 1.3-1.35 GPa for temperatures between 80 and $100 \mathrm{~K}$ [50,51]. Experimentally, it is observed at $1-1.1 \mathrm{GPa}$ in this temperature range. Also, the completely filled clathrates, with stoichiometries $\mathrm{Ng} \cdot 5.75 \mathrm{H}_{2} \mathrm{O}(\mathrm{CS}-\mathrm{I})$ and $\mathrm{Ng} \cdot 5.67 \mathrm{H}_{2} \mathrm{O}$ (CS-II), show a strikingly similar behavior irrespective of the kind of cubic structure. With increasing size of $\mathrm{Ng}$, the amorphization pressure increases as $1.5 \mathrm{GPa}(\mathrm{Ne}), 1.6 \mathrm{GPa}(\mathrm{Ar})$, and $1.9 \mathrm{GPa}$ $(\mathrm{Kr})$, with the peculiarity for Xe discussed in the previous section. Note that, for this generalized PIA discussion, we generically refer to a discontinuous density change as "amorphization," as we did not analyze Kr hydrate and the partially filled model systems discussed below for a semicrystalline state.

To find out about the influence of the different cages, we next address different compositions in model systems referring to either fully occupied large ( $\mathrm{T}$ and $\mathrm{H}$ ) cages or fully occupied small (D) cages. If only the six $\mathrm{T}$ cages are occupied in CS-I, the composition is $\mathrm{Ng} \cdot 7.67 \mathrm{H}_{2} \mathrm{O}$. If only the two D cages are filled, the composition is $\mathrm{Ng} \cdot 23 \mathrm{H}_{2} \mathrm{O}$. With only $\mathrm{H}$ cages occupied, the composition for a CS-II (a)

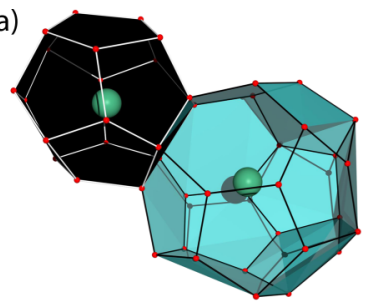

(b)

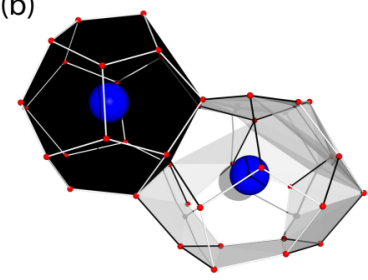

(c)
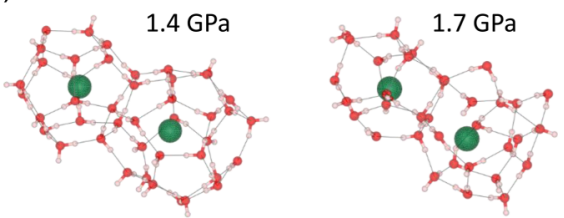

(d)
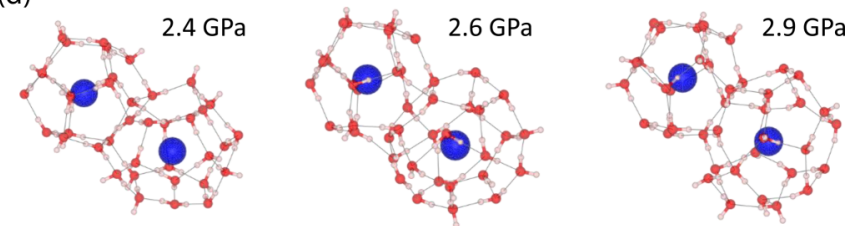

FIG. 9. Molecular dynamics (MD) snapshots showing the major structural features of Ar and Xe hydrate prior and after discontinuous densification at $95 \mathrm{~K}$. (a) Crystalline CS-II Ar hydrate at $1.4 \mathrm{GPa}$. (b) Crystalline CS-I Xe hydrate at $2.0 \mathrm{GPa}$. (c) Guest environments in Ar hydrate at $1.4 \mathrm{GPa}$ (crystalline) and $1.7 \mathrm{GPa}$ (amorphous). (d) Guest environments in Xe hydrate at $2.4 \mathrm{GPa}$ (crystalline) and 2.6 and $2.9 \mathrm{GPa}$ (semicrystalline with disordered large cages). Red spheres: O, blue: Xe, green: Ar, white: $\mathrm{H}$.

clathrate is $\mathrm{Ng} \cdot 17 \mathrm{H}_{2} \mathrm{O}$, which is experimentally observed for, e.g., THF hydrate. If only D cages are occupied, then the composition is $\mathrm{Ng} \cdot 8.5 \mathrm{H}_{2} \mathrm{O}$. CS-II Ne hydrate represents a special case since it assumes a more guest-rich composition of $\mathrm{Ng} \cdot 4.25 \mathrm{H}_{2} \mathrm{O}$ with doubly occupied $\mathrm{H}$ cages [52], and this scenario is also included in our investigation.

The results are shown in Fig. 11. CS-I possesses a larger fraction of large cages $\mathrm{T}$ than CS-II. Only filling of minority cages stabilizes CS-I marginally, by $0.1 \mathrm{GPa}$ for $\mathrm{Ar}$ and $\mathrm{Kr}$ and 0.2 GPa for (oversized) Xe [Fig. 11(a)]. A larger effect of the increasing size of $\mathrm{Ng}$ is seen when filling majority $\mathrm{T}$ cages. The amorphization pressure increases from 1.5 GPa (Ne) to $1.55 \mathrm{GPa}(\mathrm{Ar})$ to $1.65 \mathrm{GPa}(\mathrm{Kr})$ to $2 \mathrm{GPa}$ for $\mathrm{Xe}$. The stability of fully occupied CS-I [cf. Fig. 10(a)] is a cooperative effect of both $\mathrm{T}$ and $\mathrm{D}$ cage filling. CS-II possesses a larger fraction of small D cages than CS-I. Filling of minority $\mathrm{H}$ cages has no stabilizing effect with respect to empty CS-II, i.e., ice XVI [53], irrespective of the size of the $\mathrm{Ng}$ [Fig. 11(b)]. This agrees with the observation that, for CS-II clathrates, with rather large organic molecules, such as THF, 1,3-dioxolane, and cyclobutanone, for which only the large $\mathrm{H}$ cages are filled, the amorphization behavior is virtually identical $[27,49]$. Filling of the majority D cages has a clear stabilizing effect, which depends pronouncedly on the size of the $\mathrm{Ng}$. The amorphization pressure is shifted to $1.5 \mathrm{GPa}$ for $\mathrm{Ne}$, to $1.6 \mathrm{GPa}$ for $\mathrm{Ar}$, to $1.8 \mathrm{GPa}$ for $\mathrm{Kr}$, which is closely mimicking the sequence of PIA pressures for the fully occupied case (Fig. 10). This indicates that small or majority cage filling almost solely determines the stability of CS-II hydrates with 

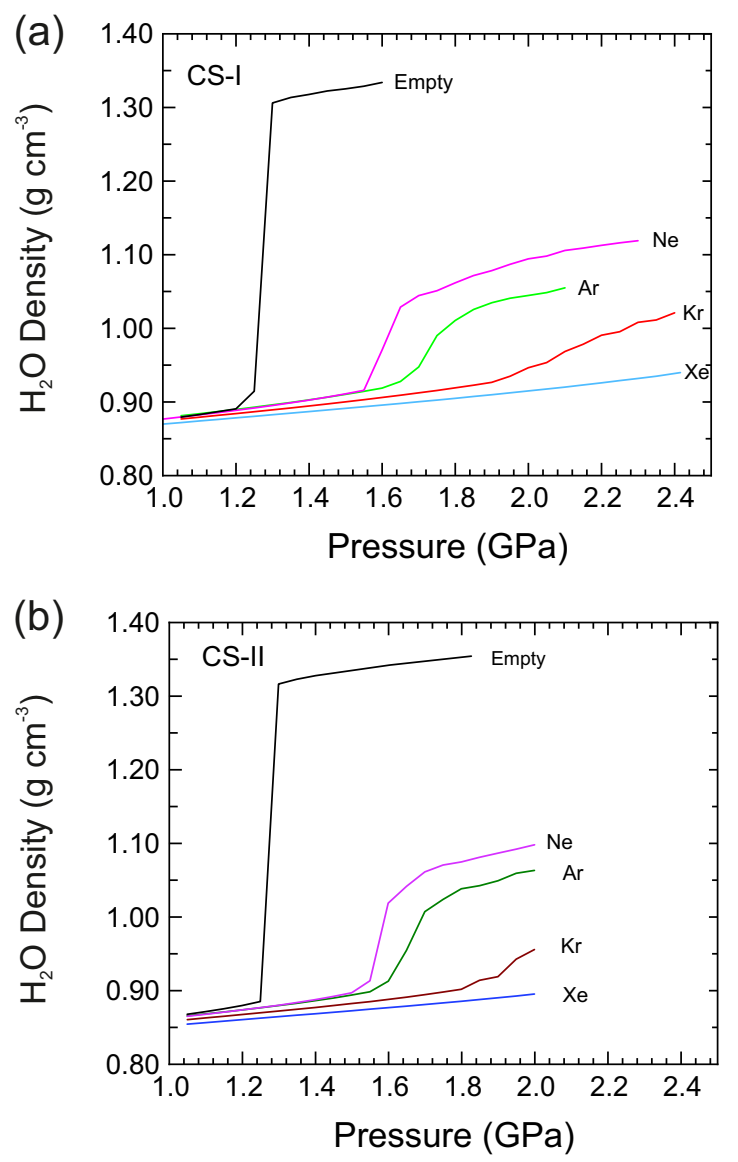

FIG. 10. Molecular dynamics (MD) derived density-pressure relations for (a) CS-I and (b) CS-II noble gas clathrate hydrates at $95 \mathrm{~K}$. Both types of cages in each structure are completely filled, i.e., the compositions correspond to $\mathrm{Ng} \cdot 5.75 \mathrm{H}_{2} \mathrm{O}$ for CS-I and $\mathrm{Ng} \cdot 5.67 \mathrm{H}_{2} \mathrm{O}$ for CS-II.

respect to amorphization. The Ne case of doubly occupied $\mathrm{H}$ cages coherently mimics the behavior of a twice-as-large $\mathrm{Ng}\left(\mathrm{Ne} V_{\mathrm{VdW}}=15 \AA^{3}, \mathrm{Ar} V_{\mathrm{VdW}}=28 \AA^{3}, \mathrm{Kr} V_{\mathrm{VdW}}=35 \AA^{3}\right.$, $\mathrm{Xe} V_{\mathrm{VdW}}=42 \AA^{3}$ ) [48], with a collapse pressure at $1.7 \mathrm{GPa}$ which is between that of Ar and $\mathrm{Kr}$ hydrate. Similarly, when $\mathrm{D}$ cages are empty and $\mathrm{H}$ cages remain doubly occupied, only a minor stabilization effect is observed.

\section{CONCLUSIONS}

The high-pressure structural behavior of $\mathrm{Ng}$ clathrate hydrates was investigated at $95 \mathrm{~K}$. As a general phenomenon, $\mathrm{Ng}$ clathrate hydrates undergo PIA at low temperatures, whereas at temperatures close to room temperature, crystal-crystal transitions to more guest-rich hydrate phases are observed [8-10,14,15,25]. Particularly, NPD experiments show that CS-II AH and CS-I XH amorphize at 1.4 and above 4 GPa, respectively. From MD simulations, it was concluded that, without a guest species, both cubic clathrate structures amorphize at $1.2 \mathrm{GPa}$, which is very similar to hexagonal ice. Filling of large-sized $\mathrm{H}$ cages does not provide stability toward amorphization for structure II, whereas filled small-sized dodecahedral D cages shift PIA to successively higher pressures with increasing size of the $\mathrm{Ng}$ guest. For CS-I, filling
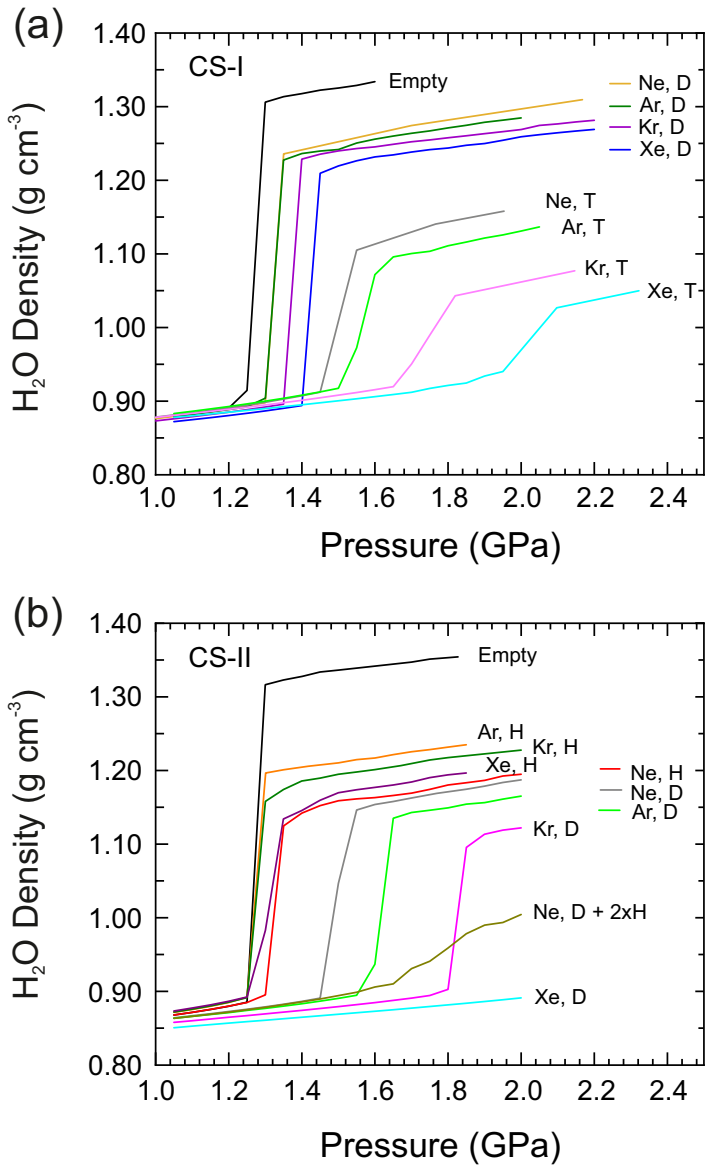

FIG. 11. Molecular dynamics (MD) derived density-pressure relations for CS-I and CS-II noble gas clathrate hydrates at $95 \mathrm{~K}$ considering different cage fillings. (a) Empty CS-I and Ne-Xe hydrate with only T or only D cages filled. (b) Empty CS-II (ice XVI) and $\mathrm{Ne}-\mathrm{Xe}$ hydrate with only $\mathrm{D}$ or only $\mathrm{H}$ cages filled. CS-II $\mathrm{Ne}$ hydrate with only $\mathrm{H}$ cages doubly occupied (red line) and $\mathrm{D}$ cages singly occupied and $\mathrm{H}$ cages doubly occupied (olive line).

of both kinds of cages, large-sized T and small-sized D, acts to stabilize in a cooperative fashion. When fully occupied (referring to compositions $\mathrm{Ng} \cdot 5.75 \mathrm{H}_{2} \mathrm{O}$ and $\mathrm{Ng} \cdot 5.67 \mathrm{H}_{2} \mathrm{O}$ for CS-I and II, respectively), both cubic structures exhibit a similar behavior with PIA occurring in the pressure range of 1.3-2 GPa, depending on the size of the Ng. Xe represents a special case because it appears oversized as guest. Xe hydrate transforms at $2.5 \mathrm{GPa}$ to a semicrystalline state in which the long-range order of $\mathrm{Xe}$ atoms and their water coordination numbers from the crystalline state are essentially retained. Continuous amorphization takes place above $3 \mathrm{GPa}$. We conjecture the similarity of Xe atom positions in the crystalline and amorphous state is the reason why amorphous $\mathrm{XH}$ recrystallized upon decompression in all recovery attempts. In contrast, amorphous CS-II AH is recoverable at ambient pressure when annealed at $>1.5 \mathrm{GPa}$ and $170 \mathrm{~K}$.

\section{ACKNOWLEDGMENTS}

This research has been funded by the Swedish Foundation for Strategic Research (SSF) within the Swedish 
national graduate school in neutron scattering (SwedNess). A portion of this research used resources at the Spallation Neutron Source, a DOE Office of Science User Facility operated by the Oak Ridge National Laboratory. The simulations were performed on resources provided by the Swedish National Infrastructure for Computing (SNIC) at the National Supercomputer Centre (NSC). The authors also thank the financial support from eSSENCE, the Swedish Foundation for International Cooperation in Research and Higher Education (STINT), the Brazilian agency CAPES (Project CAPES/STINT $\mathrm{N}^{\circ} 88887.304724 / 2018$ ) and the Swedish Research Council (Registration No. 2019-05366).
[1] E. D. Sloan Jr. and C. A. Koh, Clathrate Hydrates of Natural Gases, (CRC Press, Boca Raton, 2007).

[2] J. H. van der Waals and J. C. Platteeuw, Clathrate solutions, in Advances in Chemical Physics, edited by I. Prigogine (John Wiley \& Sons, Ltd, New Jersey, 2007).

[3] G. A. Jeffrey, Hydrate inclusion compounds, J. Incl. Phenom. 1, 211 (1984).

[4] R. M. Barrer and W. I. Stuart, Non-stoicheiometric clathrate compounds of water, Proc. R. Soc. A 243, 172 (1957).

[5] R. K. McMullan and G. A. Jeffrey, Polyhedral clathrate hydrates. IX. structure of ethylene oxide hydrate, J. Chem. Phys. 42, 2725 (1965).

[6] P. H. B. Brant Carvalho, A. Mace, O. Andersson, C. A. Tulk, J. Molaison, and U. Häussermann, Elucidating the guest disorder in structure II argon hydrate-a neutron diffraction isotopic substitution study, J. Solid State Chem. 285, 121220 (2020)

[7] C. A. Koh, Towards a fundamental understanding of natural gas hydrates, Chem. Soc. Rev. 31, 157 (2002).

[8] A. Y. Manakov, V. I. Voronin, A. V. Kurnosov, A. E. Teplykh, V. Y. Komarov, and Y. A. Dyadin, Structural investigations of argon hydrates at pressures up to $10 \mathrm{kbar}$, J. Incl. Phenom. 48, 11 (2004)

[9] J. S. Loveday and R. J. Nelmes, High-pressure gas hydrates, Phys. Chem. Chem. Phys. 10, 937 (2008).

[10] A. Y. Manakov, A. Y. Likhacheva, V. A. Potemkin, A. G. Ogienko, A. V. Kurnosov, and A. I. Ancharov, Compressibility of gas hydrates, Chem. Phys. Chem. 12, 2476 (2011).

[11] G. G. Malenkov, Argon and water, J. Struct. Chem. 54, 252 (2013).

[12] L. Yang, C. A. Tulk, D. D. Klug, I. L. Moudrakovski, C. I. Ratcliffe, J. A. Ripmeester, B. C. Chakoumakos, L. Ehmd, C. D. Martin, and J. B. Parise, Synthesis and characterization of a new structure of gas hydrate, Proc. Natl. Acad. Sci. USA 106, 6060 (2009).

[13] Y. A. Dyadin, Clathrate formation in $\mathrm{Kr}-\mathrm{H}_{2} \mathrm{O}$ and $\mathrm{Xe}-\mathrm{H}_{2} \mathrm{O}$ systems under pressures up to $15 \mathrm{kbar}$, Mendeleev Commun. 7, 74 (1997).

[14] Y. A. Dyadin, Clathrate hydrate of xenon at high pressure, Mendeleev Commun. 6, 44 (1996).

[15] C. Sanloup, H. K. Mao, and R. J. Hemley, High-pressure transformations in xenon hydrates, Proc. Natl. Acad. Sci. USA 99, 25 (2002).

[16] A. Y. Manakov, V. I. Kosyakov, and S. F. Solodovnikov, Structural chemistry of clathrate hydrates and related compounds, in Comprehensive Supramolecular Chemistry II (Elsevier Inc., Amsterdam, 2017), Vol. 7, pp. 161-206.

[17] Y. F. Makogon, Natural gas hydrates-a promising source of energy, J. Nat. Gas Sci. Eng. 2, 49 (2010).
[18] T. B. Peters, J. L. Smith, and J. G. Brisson, Production of $\mathrm{CO}_{2}$ clathrate hydrate frozen desserts by flash freezing, J. Food Eng. 100, 669 (2010).

[19] X. Peng, Y. Hu, Y. Liu, C. Jin, and H. Lin, Separation of ionic liquids from dilute aqueous solutions using the method based on $\mathrm{CO}_{2}$ hydrates, J. Nat. Gas Chem. 19, 81 (2010).

[20] A. G. Ogienko, E. V. Boldyreva, A. Y. Manakov, S. A. Myz, A. A. Ogienko, A. S. Yunoshev, E. G. Zevak, N. V. Kutaev, and A. A. Krasnikov, Preparation of fine powders of pharmaceutical substances by freeze-drying of frozen solutions in systems with clathrate formation, Dokl. Phys. Chem. 444, 88 (2012).

[21] A. Hassanpouryouzband, E. Joonaki, M. Vasheghani Farahani, S. Takeya, C. Ruppel, J. Yang, N. J. English, J. M. Schicks, K. Edlmann, H. Mehrabian, Z. M. Aman, and B. Tohidi, Gas hydrates in sustainable chemistry, Chem. Soc. Rev. 49, 5225 (2020).

[22] W. L. Mao, C. A. Koh, and E. D. Sloan, Clathrate hydrates under pressure, Phys. Today 60, 42 (2007).

[23] R. Flacau, S. Desgreniers, and J. S. Tse, Electron density topology of cubic structure i Xe clathrate hydrate at high pressure, J. Chem. Phys. 129, 244507 (2008).

[24] L. Yang, C. A. Tulk, D. D. Klug, B. C. Chakoumakos, L. Ehm, J. J. Molaison, J. B. Parise, and J. M. Simonson, Guest disorder and high pressure behavior of argon hydrates, Chem. Phys. Lett. 485, 104 (2010).

[25] Y. A. Dyadin, E. G. Larionov, D. S. Mirinskij, T. V. Mikina, E. Y. Aladko, and L. I. Starostina, Phase diagram of the $\mathrm{Xe}^{-\mathrm{H}_{2} \mathrm{O}}$ system up to 15 kbar, J. Incl. Phenom. Mol. Recognit. Chem. 28, 271 (1997).

[26] O. Mishima, Relationship between melting and amorphization of ice, Nature 384, 546 (1996).

[27] Y. P. Handa, J. S. Tse, D. D. Klug, and E. Whalley, Pressureinduced phase transitions in clathrate hydrates, J. Chem. Phys. 94, 623 (1991).

[28] H. Tanaka and Y. Amano, Pressure-induced amorphization of clathrate hydrates, Mol. Phys. 100, 2183 (2002).

[29] See Supplemental Material at http://link.aps.org/supplemental/ 10.1103/PhysRevB.103.064205 for a description in detail of the neutron diffraction experiments and extra figures.

[30] M. Matsumoto, T. Yagasaki, and H. Tanaka, GenIce: hydrogendisordered ice generator, J. Comput. Chem. 39, 61 (2018).

[31] S. Plimpton, Fast parallel algorithms for short-range molecular dynamics, J. Comput. Phys. 117, 1 (1995), See http://lammps. sandia.gov for information about the LAMMPS software.

[32] J. L. F. Abascal, E. Sanz, R. G. Fernández, and C. Vega, A potential model for the study of ices and amorphous water: TIP4P/ice, J. Chem. Phys. 122, 234511 (2005).

[33] A. K. Rappé, C. J. Casewit, K. S. Colwell, W. A. Goddard, and W. M. Skiff, UFF, a full periodic table force field for molecular 
mechanics and molecular dynamics simulations, J. Am. Chem. Soc. 114, 10024 (1992).

[34] S. Melchionna, G. Ciccotti, and B. L. Holian, Hoover NPT dynamics for systems varying in shape and size, Mol. Phys. 78, 533 (1993).

[35] V. S. Neverov, XaNSoNS: GPU-accelerated simulator of diffraction patterns of nanoparticles, SoftwareX 6, 63 (2017).

[36] T. Ikeda-Fukazawa, Y. Yamaguchi, K. Nagashima, and K. Kawamura, Structure and dynamics of empty cages in xenon clathrate hydrate, J. Chem. Phys. 129, 224506 (2008).

[37] O. Andersson, P. H. B. Brant Carvalho, Y. J. Hsu, and U. Häussermann, Transitions in pressure-amorphized clathrate hydrates akin to those of amorphous ices, J. Chem. Phys. 151, 014502 (2019).

[38] Y. Suzuki, Evidence of pressure-induced amorphization of tetrahydrofuran clathrate hydrate, Phys. Rev. B 70, 172108 (2004).

[39] M. Bauer, D. M. Többens, E. Mayer, and T. Loerting, Pressureamorphized cubic structure II clathrate hydrate: Crystallization in slow motion, Phys. Chem. Chem. Phys. 13, 2167 (2011).

[40] O. Andersson and G. P. Johari, Nature of the pressure-induced collapse of an ice clathrate by dielectric spectroscopy, J. Chem. Phys. 129, 234505 (2008).

[41] O. Andersson and Y. Nakazawa, Transitions in pressure collapsed clathrate hydrates, J. Phys. Chem. B 119, 3846 (2015).

[42] T. Loerting, C. Salzmann, I. Kohl, E. Mayer, and A. Hallbrucker, A second distinct structural 'state' of high-density amorphous ice at $77 \mathrm{~K}$ and 1 bar, Phys. Chem. Chem. Phys. 3, 5355 (2001).

[43] R. M. Barrer and A. V. J. Edge, Gas hydrates containing argon, krypton and xenon: kinetics and energetics of formation and equilibria, Proc. R. Soc. A 300, 1 (1967).

[44] S. Klotz, G. Hamel, J. S. Loveday, R. J. Nelmes, and M. Guthrie, Recrystallisation of HDA ice under pressure by in-situ neutron diffraction to $3.9 \mathrm{GPa}$, Z. Kristallogr. 218, 117 (2003).
[45] J. R. MacDonald, Review of some experimental and analytical equations of state, Rev. Mod. Phys. 41, 316 (1969).

[46] V. Vinš, A. Jäger, S. Hielscher, R. Span,, J. Hrubý, and C. Breitkopf, Temperature \& pressure correlation for volume of gas hydrates with crystal structures sI \& sII, in EPJ Web of Conferences, edited by P. Dančová (EDP Sciences, Saint Louis, 2017), Vol. 143, p. 02141.

[47] A. Y. Manakov, A. G. Ogienko, M. Tkacz, J. Lipkowski, A. S. Stoporev, and N. V. Kutaev, High-pressure gas hydrates of argon: Compositions and equations of state, J. Phys. Chem. B 115, 9564 (2011).

[48] A. Bondi, van der Waals Volumes and Radii, J. Phys. Chem. 68, 441 (1964).

[49] P. H. B. Brant Carvalho, A. Mace, C. L. Bull, N. P. Funnell, C. A. Tulk, O. Andersson, and U. Häussermann, Elucidation of the pressure induced amorphization of tetrahydrofuran clathrate hydrate, J. Chem. Phys. 150, 204506 (2019).

[50] F. Martelli, N. Giovambattista, S. Torquato, and R. Car, Searching for crystal-ice domains in amorphous ices, Phys. Rev. Mater. 2, 075601 (2018).

[51] J. Engstler and N. Giovambattista, Heating- and pressureinduced transformations in amorphous and hexagonal ice: A computer simulation study using the TIP4P/2005 model, J. Chem. Phys. 147, 74505 (2017).

[52] K. A. Lokshin, Y. Zhao, D. He, W. L. Mao, H. K. Mao, R. J. Hemley, M. V. Lobanov, and M. Greenblatt, Structure and Dynamics of Hydrogen Molecules in the Novel Clathrate Hydrate by High Pressure Neutron Diffraction, Phys. Rev. Lett. 93, 125503 (2004).

[53] A. Falenty, T. C. Hansen, and W. F. Kuhs, Formation and properties of ice XVI obtained by emptying a type sII clathrate hydrate, Nature 516, 231 (2014).

[54] C. G. Salzmann, Advances in the experimental exploration of water's phase diagram, J. Chem. Phys. 150, 060901 (2019). 